\title{
Transitional interventions to reduce early psychiatric readmissions in adults: systematic review
}

Simone N. Vigod, Paul A. Kurdyak, Cindy-Lee Dennis, Talia Leszcz, Valerie H. Taylor, Daniel M. Blumberger and Dallas P. Seitz

\section{Background}

Up to $13 \%$ of psychiatric patients are readmitted shortly after discharge. Interventions that ensure successful transitions to community care may play a key role in preventing early readmission.

\begin{abstract}
Aims
To describe and evaluate interventions applied during the transition from in-patient to out-patient care in preventing early psychiatric readmission.
\end{abstract}

\section{Method}

Systematic review of transitional interventions among adults admitted to hospital with mental illness where the study outcome was psychiatric readmission.

\section{Results}

The review included 15 studies with 15 non-overlapping intervention components. Absolute risk reductions of 13.6 to $37.0 \%$ were observed in statistically significant studies. Effective intervention components were: pre- and postdischarge patient psychoeducation, structured needs assessments, medication reconciliation/education, transition managers and in-patient/out-patient provider communication. Key limitations were small sample size and risk of bias.

\section{Conclusions}

Many effective transitional intervention components are feasible and likely to be cost-effective. Future research can provide direction about the specific components necessary and/or sufficient for preventing early psychiatric readmission.

\section{Declaration of interest}

S.N.V. was supported by a research training fellowship from the Ontario Mental Health Foundation and by a Clinician-Scientist award at the Women's College Research Institute during the development of this study. She received a one-time consulting fee from Multi-Dimensional Health Care $(\mathrm{MDH})$ consulting for the development of continuing healthcare activities related to perinatal mental health. D.M.B. receives research support from Brainsway Ltd for an investigator-initiated study. V.H.T. receives funding from Bristol-Myers Squibb for an investigator-initiated study and has been a speaker for AstraZeneca, Bristol-Myers Squibb, Eli Lilly and Lundbeck.
Data from high-income countries including the UK, USA and Canada report that up to $13 \%$ of psychiatric patients are readmitted shortly after discharge from acute psychiatric units. ${ }^{1-4}$ Early readmission, usually defined as within 90 days of discharge, represents a negative clinical outcome for patients. Given the high cost of emergency department visits and in-patient psychiatric treatment, it is an important economic issue for policy-makers as well. As such, early psychiatric readmission has been adopted as a negative quality of care indicator internationally and governments are now setting benchmarks for reducing early readmission. ${ }^{1,5,6}$ In targeting this problem, a key issue that has been explored is that early psychiatric readmission may reflect not only the quality of in-patient care, ${ }^{1,7}$ but also the degree of continuity of care with services provided in other parts of the mental health system. ${ }^{8}$ In particular, it may reflect the ability of mental health systems to provide coordinated care and support as patients transition from hospital to less intensive types of ambulatory care. ${ }^{1}$ Surprisingly, however, evidence in support of interventions designed to optimise this transition is limited. Reports about quality improvement initiatives exist; ${ }^{9}$ but a systematic review evaluating the role of pre-discharge interventions in reducing psychiatric readmission identified only four studies in the literature. This review was limited by its restriction to interventions that occurred prior to discharge and the authors were not able to comment on the necessary and/or sufficient components for the effectiveness of any of the included interventions. ${ }^{10}$ As such, the purpose of our systematic review was to describe and evaluate the efficacy of interventions designed to improve the transition from in-patient to out-patient care in preventing early psychiatric readmission among adults with mental illness.

\section{Method}

\section{Overview}

We performed a systematic review of interventions whose goals were to assist in the successful transition from in-patient to out-patient care for adult in-patients on psychiatric units. Interventions were implemented during the in-patient admission, in the early post-discharge period or spanned the transition from in-patient to out-patient care (i.e. bridging interventions). ${ }^{11-13} \mathrm{We}$ sought to describe and categorise published interventions and assess their efficacy in reducing the risk of early readmission.

\section{Search strategy}

We searched the following electronic databases from inception until January 2012 using medical subject heading and keywords: Medline, CINAHL, EMBASE, PsycINFO and the Cochrane Library. The following terms were developed in collaboration with a hospital research librarian and used to identify potentially relevant articles: Hospitalization, Recurrence, post discharge, postdischarge, rehospitali ${ }^{*}$ readmit ${ }^{\star}$, discharge planning, continuity of patient care, co-ordination, coordination, outpatient-care, ambulatory-care, transitional-care, aftercare, in combination with mental, psychiatr ${ }^{*}$, mental disorders, mentally ill persons, mental health services AND intervention ${ }^{*}$, therapy. The preliminary database search was conducted by one author 
(T.L.). Bibliographies of articles and review articles were handsearched to identify primary articles that may have been missed in the initial search. Only published, peer-reviewed articles available in English were included. Peer-reviewed publications were identified in the initial stage of the search process, and potentially relevant abstracts meeting the predetermined eligibility criteria were retrieved to review for inclusion criteria.

\section{Criteria for study selection}

\section{Inclusion criteria}

We included interventions whose goals were to assist in the transition from in-patient to out-patient care for adult in-patients on psychiatric units. Eligible interventions could have components delivered prior to discharge (pre-discharge), shortly after discharge (post-discharge) or could span both the pre- and post-discharge time periods (bridging). ${ }^{11-13}$ We did not restrict studies based on the duration of evaluated interventions. Studies evaluating intensive case management programmes (such as assertive community treatment) were eligible for inclusion because difficulties in the transition to out-patient care may occur even in such a setting. However, we required that such studies specifically evaluate a transitional intervention component. Psychiatric readmission after hospital discharge was a required outcome in all studies. Where studies reported readmission rates at multiple time points, we selected the period of time closest to the discharge date as the primary outcome. Studies of any design were included provided that outcomes were reported in an intervention group and non-intervention group.

\section{Exclusion criteria}

We excluded interventions not related to adult mental health (i.e. medical, surgical, obstetric, paediatric population), interventions conducted exclusively in populations with substance use disorders and interventions that were involuntary in nature (for example, involuntary treatment orders, legal or forensic interventions). We excluded interventions directed at treating specific psychiatric disorders (for example, using medication or specific psychotherapies) unless there was a component of the intervention specifically aimed at improving the transition from in-patient to out-patient care.

\section{Data extraction and classification}

Two authors (S.N.V. and T.L.) independently extracted the data from all studies and the co-authors each independently extracted data from a subset of the articles into data summary tables. Data on the study populations, study design, intervention description, outcome description, timing of measurement(s) and outcome rates in the intervention and comparison groups were extracted from each included study. Because most interventions were complex and involved multiple components, the principal author (S.N.V.) then categorised the components of each intervention according to whether the components were implemented pre-discharge, post-discharge or bridged the transition from in-patient to out-patient care (i.e. bridging components). These categorisations were reviewed with all of the other co-authors until a consensus was reached that a set of non-overlapping components had been developed to reflect the intervention strategies described in the included studies.

\section{Quality assessment}

To assess internal validity of the studies, each study was examined for risk of bias using a standard form adapted from the Cochrane
Effective Practice and Organisation of Care (EPOC) group's risk of bias criteria. ${ }^{14}$ Specifically, risk of bias in each of the individual studies was assessed using the nine EPOC criteria: randomisation, allocation concealment, baseline differences in outcome measurement, baseline differences in any known or potentially unknown differences between groups that might influence prognosis, treatment of missing data, objectivity of outcome assessment, risk of contamination, risk of selective outcome reporting and other sources of bias (for example related to sample size, design, analytical flaws). The baseline differences considered important to evaluate for this review included sociodemographic, medical and psychiatric characteristics, such as: low income, poor social support, medical comorbidity and number of previous mental health admissions. ${ }^{1,7}$ Included articles were systematically evaluated for whether they reported on these potential confounding factors. One author (S.N.V.) performed a risk of bias assessment for all studies along with one additional co-author, such that each article received two independent assessments. Disagreements between reviewers were resolved by consensus, with a third reviewer consulted if agreement could not be reached.

\section{Data analysis}

The characteristics of studies and attributes of study quality were summarised in tables. Studies were categorised chronologically and by study design (for example randomised controlled trial (RCT) and other study designs). The intervention components within studies were categorised as pre-discharge, post-discharge or bridging. For each study, we calculated absolute risk reduction in readmission rates (in percentage points) or days in hospital during the follow-up period (in days) for each intervention. We planned to combine data quantitatively across studies using standard meta-analytic techniques to arrive at pooled estimates of the odds ratios for readmission associated with interventions compared with usual care where there were a sufficient number of clinical homogeneous studies.

\section{Results}

\section{Characteristics of included studies}

Database searches yielded 477 unique articles. Reference lists of relevant articles and of 14 relevant systematic reviews were reviewed in an attempt to identify any additional articles meeting inclusion criteria $(n=5)$. In total, 105 full-text articles were retrieved and reviewed in detail, with 15 meeting inclusion criteria (Fig. 1). ${ }^{15}$

All included studies were conducted in high-income countries, with the majority from the USA. ${ }^{16-30}$ There were eight RCTs, five controlled clinical trials and two cohort studies. Just over half of included studies (8 out of 15) restricted their samples to schizophrenia or schizoaffective disorder diagnoses, whereas the others included more representative samples of in-patients. Although most studies excluded participants who were already enrolled in case management programmes, the baseline admission history of participants varied substantially across studies (for example, mean number of previous admissions ranged from 1.9 to 5.8) (see online Table DS1).

\section{Classification and description of intervention components}

We identified a set of 15 non-overlapping intervention components within the included studies. These included four pre-discharge, eight post-discharge and three bridging intervention components (Table 1). Six studies allowed for the 


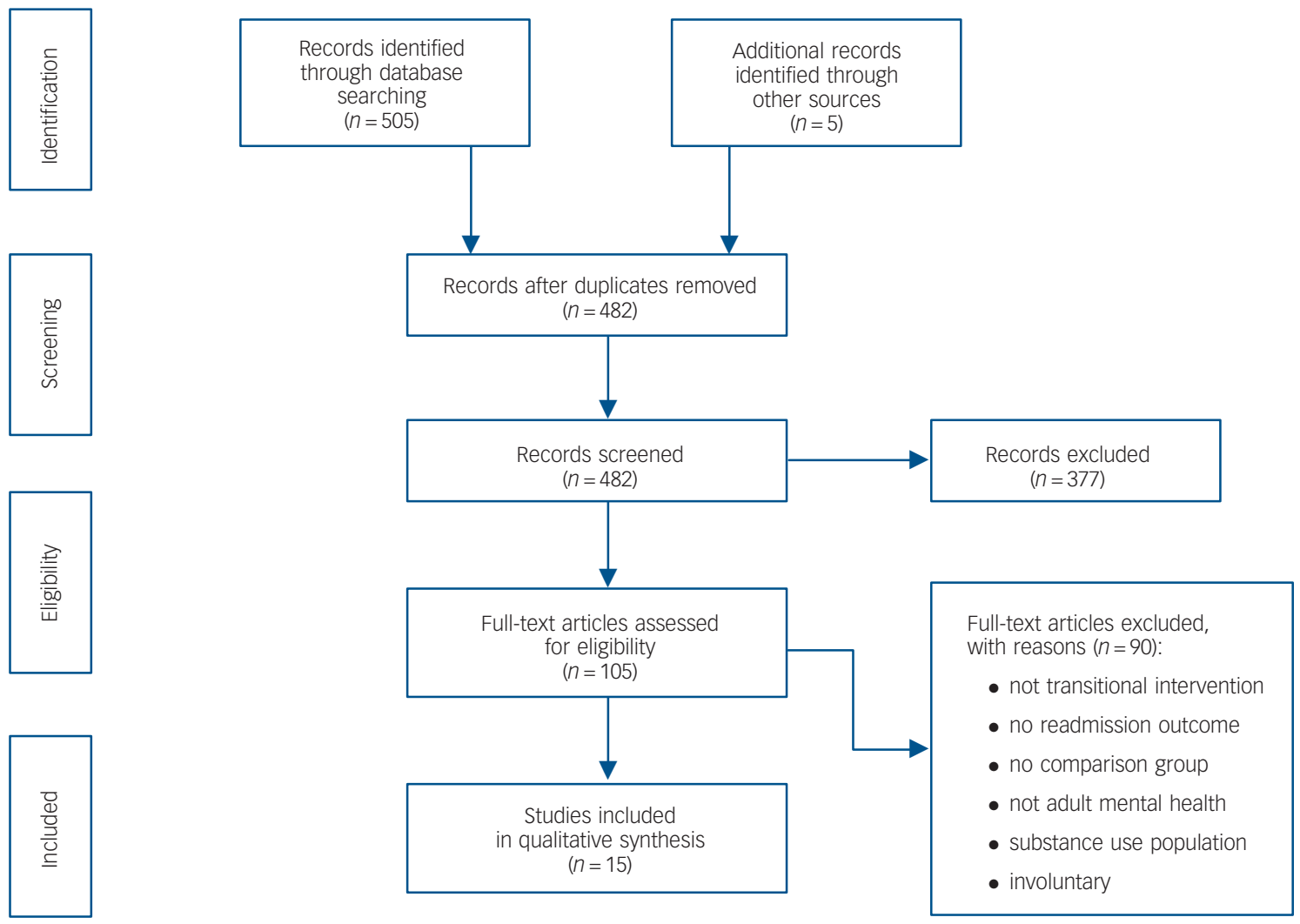

Fig. 1 Preferred Reporting Items for Systematic Reviews and Meta-analyses (PRISMA) flow diagram. ${ }^{15}$

evaluation of distinct intervention components. ${ }^{16-22}$ The number of intervention components in the other studies ranged from four to nine components. Multicomponent interventions were often developed explicitly as transitional models of care that included multiple pre-discharge, post-discharge and bridging strategies/ components.

\section{Pre-discharge components}

Seven studies tested interventions with pre-discharge components. Two studies evaluated a pre-discharge psychoeducation component derived from the fifth psychosocial module of the social and independent living skills series for individuals with schizophrenia. ${ }^{21,22}$ Three multicomponent interventions included a pre-discharge medication reconciliation/education component, ${ }^{23-25}$ three included a structured needs assessment component ${ }^{23,24,26}$ and one evaluated scheduling a follow-up appointment prior to discharge. ${ }^{27}$

\section{Post-discharge components}

Eleven studies tested interventions with post-discharge components. ${ }^{16,17,19,20,23,24,26-30}$ Post-discharge telephone followups $^{20,23,24,26-28,30}$ and home visits (usually by nurses) ${ }^{23,26,28-30}$ generally involved enquiry about mental health status and whether or not the patients had visited their out-patient mental health provider. Other components were: specific efforts to ensure timely follow-up with an out-patient care provider, ${ }^{23,24,26-28,30}$ psychoeducation, ${ }^{17,19,23,26}$ family education or communication, ${ }^{17,23,28}$ structured needs assessments, ${ }^{16,28}$ post-discharge 'hotlines' where patients could call either the in-patient ward or a case manager when in distress ${ }^{24,29}$ and peer support. ${ }^{29,30}$ Four studies allowed for assessment of distinct post-discharge intervention components: telephone follow-up; ${ }^{20}$ structured needs assessment; ${ }^{16}$ psychoeducation; ${ }^{17,19}$ and family education and communication. ${ }^{17}$ The other seven interventions involved multiple components.

\section{Bridging components}

Nine studies evaluated interventions with a bridging component. ${ }^{18,23-30}$ Bridging components included timely communication by in-patient staff with an out-patient care or community service provider after discharge, ${ }^{23-25,27,28}$ meeting with the out-patient mental health provider prior to discharge; ${ }^{18}$ and the use of a 'transition manager' who was introduced to the patient prior to discharge and after discharge to optimise the transition out of hospital. ${ }^{23,24,26,27,29,30}$ All six interventions involving a transition manager were made up of multiple postdischarge activities. In addition, four of the studies with transition managers involved pre-discharge components and three of them included another bridging component. Meeting with the outpatient provider prior to discharge was the only bridging component assessed as a distinct intervention. ${ }^{18}$

\section{Quality of included studies}

Details of the EPOC quality assessment items are detailed in online Table DS2. Most interventions were well-described, allowing a detailed review of the nature of the interventions. Of eight RCTs, three were pilot projects where the experimental group sample size was under $30^{20,21,29}$ and only two had an intervention group sample size greater than 100 participants. ${ }^{28,30}$ Seven studies reported baseline differences between groups on 


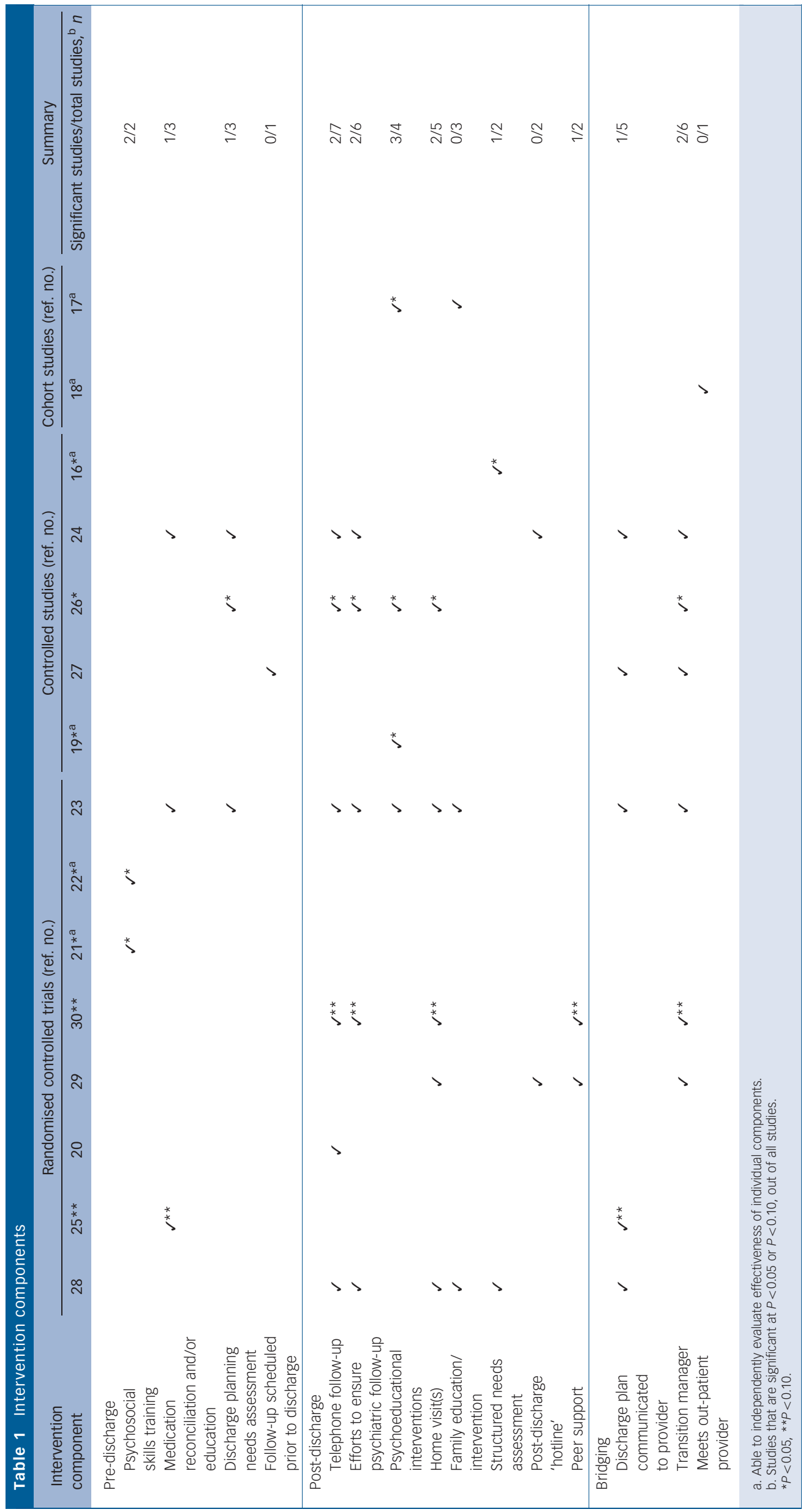


key prognostic factors for readmission such as illness severity or comorbidity that were left unaccounted for in the analysis, raising problems related to confounding in the interpretation of results. Only six studies clearly addressed issues related to individuals dropping out and missing data (nine studies either had dropout rates $>20 \%$ or did not report data in such a way that the treatment of missing data could be assessed).

\section{Intervention effects on readmission}

Although we originally planned a quantitative meta-analytic approach, we chose not to combine the data quantitatively due to substantial clinical heterogeneity. No two studies tested exactly the same intervention components and most studies had multiple intervention components. Furthermore, there was substantial variability with respect to both the timing of outcome (readmission) measurement and in readmission rates across studies (online Table DS1). In the control groups, readmission rates ranged from 13 to $36 \%$ for 3-month readmissions and from 4 to $69 \%$ for 6-24 month readmissions. For the intervention groups, the corresponding readmission rates were $7-23 \%$ for 3 -month readmissions and $0-63 \%$ for readmissions after 6 months.

\section{Interventions involving pre-discharge components}

Both studies with a pre-discharge psychoeducation intervention component generated significant results. ${ }^{21,22}$ One of three multicomponent studies involving a structured pre-discharge needs assessment component reported significant results ${ }^{26}$ and one of three multicomponent studies that involved a medication education/reconciliation component had nearly significant results. ${ }^{25}$ The study that evaluated only the scheduling of a follow-up appointment prior to discharge did not find statistically significant results. ${ }^{27}$

\section{Interventions involving post-discharge components}

Three out of four studies involving post-discharge psychoeducational components revealed significant reductions in readmission in the intervention groups. ${ }^{17,19,26}$ Two studies out of those that included telephone follow-up $(n=7)$, efforts to ensure timely follow-up $(n=6)$ and home visits $(n=5)$ had positive findings. ${ }^{26,30}$ Both interventions also included a transition manager (bridging component) as well as other pre- and/or postdischarge components. One of two studies involving a structured post-discharge needs assessment had positive findings. ${ }^{16}$ Results approached statistical significance in one of the multicomponent studies that included a peer support component. ${ }^{30}$ None of the studies assessing patient 'hotlines' or family intervention had significant findings.

\section{Interventions involving bridging components}

Two out of six studies involving a transition manager had statistically significant (or nearly significant) findings. ${ }^{26,30}$ One study involving timely communication of the discharge plan to the out-patient provider approached statistical significance. ${ }^{25}$

\section{Discussion}

\section{Summary of findings}

The purpose of this systematic review was to describe and evaluate the efficacy of interventions designed to improve the transition from in-patient to out-patient care in reducing early psychiatric readmission among adults with mental illness. The study is unique in its detailed characterisation of interventions with pre-discharge, post-discharge and bridging components and rigorous quality review. Our systematic search identified 15 studies evaluating a heterogeneous group of interventions that included 15 distinct intervention components. Interventions had a statistically significant impact on readmission in seven of the studies and the effect sizes in these studies were relatively large (13.6-37.0\% absolute reduction in readmission rates). We found evidence that, on their own, psychoeducation interventions targeting disease management and living skills reduce readmission rates, as do structured assessments of patients' discharge needs. Effective components identified within the context of multicomponent interventions were pre-discharge medication education/ reconciliation; post-discharge telephone follow-up, efforts to ensure timely follow-up appointments, home visits and peer support as well as the bridging components of transition manager and timely communication by in-patient staff with an out-patient care or community service provider during the transition.

\section{Context within existing literature}

Our results are consistent with the findings of a previous review of pre-discharge interventions in psychiatry where the pooled risk of readmission ratio for discharge planning interventions using data from only four studies was 0.66 (95\% CI $0.51-0.84$ ), suggesting that pre-discharge interventions are potentially effective, although there are a limited number of studies. ${ }^{10}$ There is a larger body of literature evaluating the delivery of ongoing community-based care for patients who are at very high risk of readmission (for example, assertive community treatment or other forms of intensive case management). Although not transitional care per se, some of the intervention components are similar to those identified in our review. In recent systematic reviews and metaanalyses comparing intensive case management to standard care, increased numbers of hospital readmissions were observed among individuals receiving intensive case management (odds ratio $(\mathrm{OR})=1.84,99 \%$ CI 1.33-2.57), ${ }^{31}$ although length of admission appears to be significantly reduced (mean difference -0.86 , CI -1.37 to -0.34$).{ }^{32}$ This likely reflects improved retention in care and enhanced quality of community care (as shown by the reduced number of days spent in hospital). None of our studies found an increased rate of readmission in the intervention group, suggesting that the interventions were not functioning to identify patients requiring urgent readmissions due to illness instability. This is perhaps not surprising considering that despite relatively high rates of repeat admissions in some of the samples that we studied, most studies explicitly excluded individuals who were receiving ongoing psychiatric case management services. Interestingly, our results contrast with a similar review of interventions to reduce 30-day readmission in medical in-patient settings where no distinct intervention component or combination of components was identified as effective in reducing early readmission rates. ${ }^{11}$ It has been argued that medical readmission may actually represent improved quality of care, potentially reflecting the fact that better hospitals keep sicker patients alive and that these patients appropriately require more readmissions. ${ }^{33-35}$ These same arguments may not be applicable to psychiatric admissions, however. Mortality is not as common an outcome for psychiatric in-patients compared with medical populations. Furthermore, major modifiable risk factors such as difficulty with medication use and lack of follow-up care explain significantly more of the variance in psychiatric readmission rates than for medical and surgical readmissions where acuity of the admission and medical comorbidity are more important predictors of readmission. ${ }^{36}$ It follows that if hospitals were to 
focus on improving the quality of care during the transition from acute psychiatric admission to ambulatory treatment, there would likely be potential to reduce psychiatric readmission rates. Although relatively few in number and of moderate quality and small sample size, the studies described in our review do suggest that this is the case.

\section{Explanations for findings}

Multiple factors may contribute to high early readmission rates. Acute psychiatric admission plays an important role in the mental healthcare system in the treatment of individuals with serious mental illness. ${ }^{37,38}$ Yet, financial pressure to reduce in-patient length of stay and realignment of mental health resources to community settings in current psychiatric practice has shifted the role of in-patient admission to stabilisation rather than complete recovery. ${ }^{37,38}$ Incomplete or partial symptomatic recovery places individuals at risk for relapse of symptoms and subsequent readmission. ${ }^{1,7}$ However, other risk factors for early readmission may be more amenable to intervention during the transition to out-patient treatment. These include medication management difficulties, impairment in self-care and absence of aftercare (i.e. referrals and timely follow-up with supports and services). ${ }^{1,7,39-43}$ Most successful interventions included in our review targeted these known major modifiable risk factors for readmission such as difficulty with medication use, knowledge about disease management and coordination of care after discharge. ${ }^{1,7,39-43}$ Interventions identified in our review that did not generate statistically significant results also targeted these same modifiable risk factors. Several of these studies may have been underpowered to detect effects because they were pilot studies or because readmission rates were not the primary outcome. However, it is notable that in two cases there were conflicting findings with regard to very similar interventions. Only one of two studies evaluating the 'transitional discharge model' and one of two studies evaluating the 'critical time intervention' had positive findings. Although the studies with the positive findings also had substantially larger sample sizes, both interventions with positive findings also had a flexible duration of transitional care lasting up to 1 year where the termination of the intervention was determined by clinical decision or information regarding successful transfer of care to a community provider. In both of the studies, the median length of the intervention was between 6 and 7 months. The corresponding negative studies had time limits of 5 months for the transitional discharge model and 3 months for the 'brief' critical time intervention. These findings underscore the potential importance of flexibility in the length of transitional care programmes.

\section{Study limitations}

Despite the promising preliminary findings of our review, our results highlight that there is a great need for additional highquality evidence to inform best practice. Given the large body of literature focused on risk factors for psychiatric readmission, ${ }^{39-43}$ it is surprising that only 15 studies evaluating transitional interventions to reduce readmission rates were identified. The majority of studies were conducted in the USA and therefore the results may not generalise to other health systems where funding and organisational characteristics may differ. Most studies had small sample sizes and were likely underpowered to detect clinically relevant effect sizes. Furthermore, although we did not directly assess the costs of interventions, only two of the included studies provided a cost-effectiveness analysis of the evaluated intervention. In addition, there were significant limitations in study quality. Few studies adequately assessed baseline differences between groups that might be important to readmission prognosis and missing data were often not adequately addressed, raising concern that either selection bias or differential loss to follow-up rates could be responsible for differences in readmission between groups. Few studies clearly demonstrated objective outcome reporting of readmissions and, even in those studies, it was unclear whether it was possible for outcomes to be captured if patients were readmitted to other institutions. Up to $20 \%$ of medical hospital readmissions are missed by failing to account for admission to other institutions and this may be an issue for mental health readmissions as well. ${ }^{44}$

The clinical heterogeneity of the studies also limits our ability to generate practice and policy implications from the data. The wide variation in readmission rates between studies suggests that these interventions were applied in populations with different levels of risk. This limits our ability to comment on how effective some of these interventions might have been in populations with different baseline levels of risk for readmission. For example, some interventions may have been applied either in populations where the control group was very well resourced and there was no additional benefit from the interventions (i.e. populations with low comparison group readmission rates) or where participant illness acuity was too severe for time-limited, focused discharge interventions to be effective. To mitigate this limitation, we provided detailed information regarding the samples in which interventions were evaluated such that knowledge-users would more readily be able to determine in which populations effective interventions might be implemented (and in which populations the said interventions have not yet been evaluated). Another limitation of the included studies is that the majority tested multiple intervention components together, making it difficult to comment about the relative contribution of any of the individual components of the interventions. This leaves unanswered questions as to what specific components of transitional care interventions are necessary to effect results. However, given the promising nature of some of these multicomponent interventions (for example critical time intervention and transitional discharge model, among others), it is likely that certain combinations of components may be more effective than the sum of their parts. A study using critical time intervention in psychiatric populations published subsequent to the submission of this manuscript for publication adds further support to this concept. ${ }^{45}$

\section{Implications}

We observed that transitional care interventions with predischarge, post-discharge and/or bridging components may reduce early psychiatric readmission - and that when they are effective, the magnitude of effect appears to be clinically meaningful. This supports the concept that decreasing early readmission rates in patients with psychiatric illness is an attainable goal. Many potentially effective intervention components described in this study, particularly patient psychoeducation, needs assessments and communication between providers, are standard components of best practice. Structured implementation of these intervention components in discharge planning might ensure that patients receive optimal quality of care, with resultant reduction in readmission risk among other positive outcomes. Other observed effective interventions do not necessarily require significant resources either, and it is feasible to evaluate them using readmission rate as one of several metrics. With such a high rate of psychiatric readmission and a paucity of high-quality evidence to inform best practice, additional 
evaluation of interventions to reduce early readmission in psychiatry is clearly needed. Policy-makers have shifted to the use of early hospital readmission as an indicator of the quality of care. Clinicians and researchers need to further develop the evidence base for clinical interventions that support more seamless transitions from in-patient to out-patient care. Future research can overcome the limitations of previous work to provide direction about specific components that are necessary and/or sufficient for preventing early psychiatric readmission. The taxonomy of classifying interventions into pre-discharge, postdischarge and bridging components may be a useful framework for better understanding which early readmissions are preventable in mental health populations and how to use transitional care interventions most effectively.

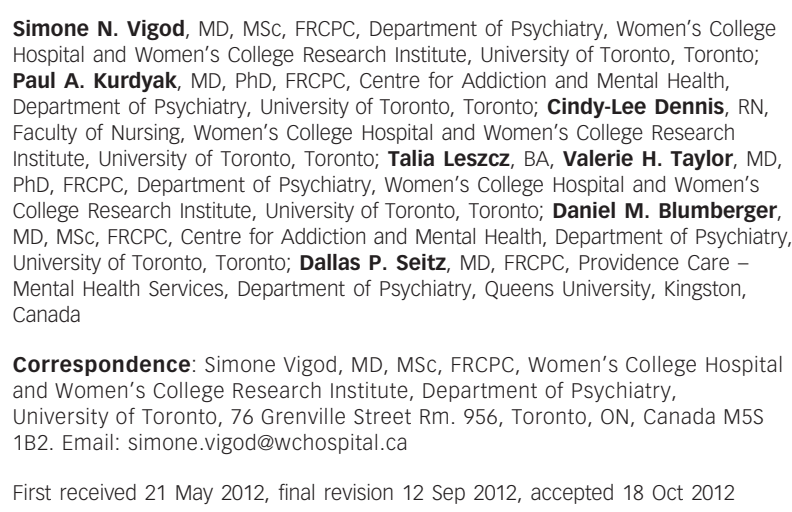

\section{References}

1 Canadian Institute for Health Information and Statistics Canada. Health Indicators 2011. ClHI, 2011.

2 Leslie DL, Rosenheck RA. Comparing quality of mental health care for public sector and privately insured populations. Psychiatr Serv 2000; 51: 650-5.

3 National Association of State Mental Health Program Directors Research Institute. 30-day Readmission Rates. National Association of State Mental Health Program Directors Research Institute, 2012.

4 Commission for Health Improvement. Psychiatric Readmissions (Adults of Working Age). Commission for Health Improvement, 2003.

5 Hermann RC, Mattke S, Somekh D, Silfverhielm H, Goldner E, Glover G, et al. Quality indicators for international benchmarking of mental health care. Int J Qual Health Care 2006; 18 (suppl 1): 31-8.

6 Rumball-Smith J, Hider $\mathrm{P}$. The validity of readmission rate as a marker of the quality of hospital care, and a recommendation for its definition. N Z Med J 2009; 122: 63-70.

7 Zhang J, Harvey C, Andrew C. Factors associated with length of stay and the risk of readmission in an acute psychiatric inpatient facility: a retrospective study. Aust N Z J Psychiatry 2011; 45: 578-85.

8 Durbin J, Lin E, Layne $\mathrm{C}$, Teed $\mathrm{M}$. Is readmission a valid indicator of the quality of inpatient psychiatric care? J Behav Health Serv Res 2007; 34: $137-50$

9 Agency for Health Care Research and Quality. Health Plan and Psychiatric Hospitals Reduce Readmissions by Reviewing Data and Developing Strategies to Improve Postdischarge Care. Agency for Health Care Research and Quality, 2012.

10 Steffen S, Kosters M, Becker T, Puschner B. Discharge planning in mental health care: a systematic review of the recent literature. Acta Psychiatr Scand 2009; 120: 1-9.

11 Hansen LO, Young RS, Hinami K, Leung A, Williams MV. Interventions to reduce 30-day rehospitalization: a systematic review. Ann Intern Med 2011; 155: $520-8$.

12 Ashton CM, Wray NP. A conceptual framework for the study of early readmission as an indicator of quality of care. Soc Sci Med 1996; 43 . 1533-41.

13 Coleman EA, Min SJ, Chomiak A, Kramer AM. Posthospital care transitions: patterns, complications, and risk identification. Health Serv Res 2004; 39 1449-65.
14 Cochrane Effective Practice and Organisation of Care Group. Risk of Bias Criteria. EPOC, no date (http://epoc.cochrane.org/sites/epoc.cochrane.org/ files/uploads/Suggested\%20risk\%20of\%20bias\%20criteria\%20for\%20EPOC \%20reviews.pdf).

15 Moher D, Liberati A, Tetzlaff J, Altman DG. Preferred reporting items for systematic reviews and meta-analyses: the PRISMA statement. PLOS Med 2009; 6: e1000097.

16 Schmidt-Kraepelin C, Janssen B, Gaebel W. Prevention of rehospitalization in schizophrenia: results of an integrated care project in Germany. Eur Arch Psychiatry Clin Neurosci 2009; 259 (S2): 205-12.

17 Prince JD. Practices preventing rehospitalization of individuals with schizophrenia. J Nerv Ment Dis 2006; 194: 397-403.

18 Olfson M, Mechanic D, Boyer CA, Hansell S. Linking inpatients with schizophrenia to outpatient care. Psychiatr Serv 1998; 49: 911-7.

19 Karniel-Lauer E, Szor H, Livne S, Melamed Y, Spiro S, Elizur A. The 're-entry group' - a transitional therapeutic framework for mentally ill patients discharged from the hospital to community clinics. Can J Psychiatry 2000; 45: 837-9.

20 Beebe L. Community nursing support for clients with schizophrenia. Arch Psychiatr Nurs 2001; 15: 214-22.

21 Wirshing DA, Pierre JM, Wirshing WC, Guzik LH, Resnick SA, Goldstein D, et al. Letter to the editors: community re-entry program training module for schizophrenic inpatients improves treatment outcomes. Schizophr Res 2006; 87: 338-9.

22 Xiang Y-T, Weng Y-Z, Li W-Y, Gao L, Chen G-L, Xie L, et al. Efficacy of the Community Re-Entry Module for patients with schizophrenia in Beijing, China: outcome at 2-year follow-up. Br J Psychiatry 2007; 190: 49-56.

23 Dixon L, Goldberg R, lannone V, Lucksted A, Brown C, Kreyenbuhl J, et al. Use of a critical time intervention to promote continuity of care after psychiatric inpatient hospitalization. Psychiatr Serv 2009; 60: 451-8.

24 Price L. Transition to community: a program to help clients with schizophrenia move from inpatient to community care: a pilot study. Arch Psychiatr Nurs 2007; 21: 336-44.

25 Shaw $\mathrm{H}$, Mackie CA, Sharkie I. Evaluation of pharmacy discharge planning on medication problems experienced by discharged acute admission mental health patients. Int J Pharm Pract 2000; 8: 144-53.

26 Kasprow WJ, Rosenheck RA. Outcomes of critical time intervention case management of homeless veterans after psychiatric hospitalization. Psychiatr Serv 2007; 58: 929-35.

27 Cuffel BJ, Held M, Goldman W. Predictive models and the effectiveness of strategies for improving outpatient follow-up under managed care. Psychiatr Serv 2002; 53: 1438-43.

28 Chiverton $\mathrm{P}$, Tortoretti $\mathrm{D}$, LaForest $\mathrm{M}$, Walker $\mathrm{PH}$. Bridging the gap between psychiatric hospitalization and community care: cost and quality outcomes. J Am Psychiatr Nurses Assoc 1999; 5: 46-53.

29 Reynolds W, Lauder W, Sharkey S, Maciver S, Cameron D. The effects of a transitional discharge model for psychiatric patients. J Psychiatr Mental Health Nurs 2004; 11: 82-4.

30 Forchuk C, Martin ML, Chan $\mathrm{YL}$, Jensen $\mathrm{E}$. Therapeutic relationships: from psychiatric hospital to community. J Psychiatr Mental Health Nurs 2005; 12: 556-64.

31 Marshall M, Gray A, Lockwood A, Green R. Case management for people with severe mental disorders. Cochrane Database Syst Rev 2000; 2: CD000050.

32 Dieterich $\mathrm{M}$, Irving $\mathrm{CB}$, Park $\mathrm{B}$, Marshall $\mathrm{M}$. Intensive case management for severe mental illness. Cochrane Database Syst Rev 2010; 10: CD007906.

33 Joynt KE, Jha AK. Thirty-day readmissions - truth and consequences. N Engl J Med 2012; 366: 1366-9.

34 van Walraven $C$, Jennings $A$, Taljaard M, Dhalla I, English S, Mulpuru S, et al. Incidence of potentially avoidable urgent readmissions and their relation to all-cause urgent readmissions. CMAJ 2011; 183: E1067-72.

35 van Walraven $C$, Bennett $C$, Jennings A, Austin PC, Forster AJ. Proportion of hospital readmissions deemed avoidable: a systematic review. CMAJ 2011; 183: E391-402.

36 van Walraven C, Dhalla IA, Bell C, Etchells E, Stiell IG, Zarnke K, et al. Derivation and validation of an index to predict early death or unplanned readmission after discharge from hospital to the community. CMAJ 2010; 182: $551-7$

37 Tulloch $A D$, Fearon $P$, David AS. Length of stay of general psychiatric inpatients in the United States: systematic review. Adm Policy Ment Health 2011; 38: 155-68.

38 Wickizer TM, Lessler D. Do treatment restrictions imposed by utilization management increase the likelihood of readmission for psychiatric patients? Med Care 1998; 36: 844-50. 
39 Lien L. Are readmission rates influenced by how psychiatric services are organized? Nord J Psychiatry 2002; 56: 23-8.

40 Nelson EA, Maruish ME, Axler JL. Effects of discharge planning and compliance with outpatient appointments on readmission rates. Psychiatr Serv 2000; 51: 885-9.

41 Oiesvold T, Saarento O, Sytema S, Vinding $\mathrm{H}$, Gostas $\mathrm{G}$, Lonnerberg $\mathrm{O}$, et al. Predictors for readmission risk of new patients: the Nordic Comparative Study on Sectorized Psychiatry. Acta Psychiatr Scand 2000; 101: 367-73.
42 Sullivan G, Wells KB, Morgenstern H, Leake B. Identifying modifiable risk factors for rehospitalization: a case-control study of seriously mentally ill persons in Mississippi. Am J Psychiatry 1995; 152: 1749-56.

43 Walker R, Minor-Schork D, Bloch R, Esinhart J. High risk factors for rehospitalization within six months. Psychiatr Q 1996; 67: 235-43.

44 Nasir $\mathrm{K}$, Lin Z, Bueno $\mathrm{H}$, Normand SL, Drye EE, Keenan PS, et al. Is samehospital readmission rate a good surrogate for all-hospital readmission rate? Med Care 2010; 48: 477-81.

45 Tomita A, Herman DB. The impact of critical time intervention in reducing psychiatric rehospitalization after hospital discharge. Psychiatr Serv 2012; 63: $935-7$.

\title{
Back Ward at Glenside
}

\section{poems by doctors}

\author{
The years are a tide on this shore \\ That has ebbed into distance \\ Leaving row after row of bedstead \\ Washed-up like driftwood \\ Where I am called in the dead of night. \\ I lock the door behind me \\ And sink into a fabulous noise \\ That rises like the keening of gulls \\ Scattered on a seaweed wind. \\ Here all our grandparents stand dribbling \\ And half naked on the smooth vinyl, \\ Staring with the tired eyes of fish. \\ Forgetting what was forgotten \\ They part as I am led to a bed in the corner. \\ For some reason the smell of pine forests \\ Thick with evening drifts from the blankets \\ Clutched by fingers bleached bone white, \\ But she is pale and pulseless now \\ With the smashed crab of nothing-to-be-done \\ From where she fell \\ Matted in her hair. \\ I fill out a form that she does not need \\ And notice in her notes a photograph \\ Faded and brown, a young woman \\ With smooth skin and a squint \\ Smiling at something \\ Beyond the camera. \\ Pigtails and lace collar dated 1920, insane. \\ Back behind the curtains \\ I lift the sheet clear of her face \\ And meet the same smile and cloudy eyes \\ Staring over my shoulder \\ To what was always out of sight, \\ Just behind this moment, \\ Her seventy lost years eddying into night.
}

Charles Montgomery, Consultant Psychiatrist, Exeter. 